\title{
IMPACTO DE UN MODELO DE INTEGRACIÓN DOCENTE ASISTENCIAL EN LA FORMACIÓN PROFESIONAL Y EL CAMPO CLÍNICO
}

\section{IMPACT OF INTEGRATION TEACHING HEALTHCARE MODEL IN THE CLINICAL FIELDS AND PROFESSIONAL FORMATION}

\author{
PAz Soto Fuentes * \\ M. Angélica García G. **
}

\begin{abstract}
RESUMEN
Los campos clínicos son un pilar fundamental en la formación de profesionales de la salud. Contar con campos clínicos pertinentes constituye una exigencia para el desarrollo de un proyecto educativo de calidad. Este estudio de corte transversal tiene como propósito evaluar el impacto en la formación y desarrollo profesionales, del modelo de integración docente asistencial desarrollado entre la Escuela de Enfermería de la Pontificia Universidad Católica de Chile y los Servicios de Enfermería de la Red de Salud UC (CIDAE-UC). Resultados: Es un modelo de trabajo que facilita el aprovechamiento de conocimientos y capacidades de enfermeras docentes y clínicas en la asistencia y la docencia, lo que se traduce en un incremento de docencia en las áreas clínicas y una mayor congruencia entre las necesidades de formación y las áreas clínicas identificadas para dar respuesta a ello. Es una estrategia de valor para preparar un personal idóneo en las diversas especialidades e incrementar la disciplina de enfermería, a través del desarrollo profesional y de la investigación colaborativa.
\end{abstract}

Palabras clave: Enfermería, servicios de integración docente asistencial, educación en enfermería.

\begin{abstract}
Clinical fields are a fundamental pillar in the training of health professionals. Counting on relevant clinical fields is a requirement for the development of an educational project of quality. This cross-sectional study aims to assess the impact in the training and on professional development of integration teaching healthcare model developed between the School of Nursing, Catholic University of Chile and the nursing services of the network of SALUD UC (CIDAE-UC). Results: working model that facilitates the use of knowledge and skills of teachers and clinical nurses in teaching and assistance, resulting in an increase in teaching in the clinical areas and a greater congruence between the needs of training and clinical areas identified to respond to this. It is a strategy of value to prepare an appropriate personnel in various specialties and increase the discipline of nursing, through professional development and collaborative research.
\end{abstract}

Key words: Nursing, teaching care integration services, nursing education.

Fecha recepción: 01/06/11 Fecha aceptación: 28/12/11

\footnotetext{
* Enfermera-matrona. Profesora asociada Escuela de Enfermería Pontificia Universidad Católica de Chile. Chile. Email: pesoto@uc.cl

${ }^{* *}$ Enfermera. Profesora asociada adjunta Escuela de Enfermería Pontificia Universidad Católica de Chile. Coordinadora docente asistencial en Enfermería EEUC/Red de Salud Universidad Católica. Chile. Email: mgarciag@uc.cl
} 


\section{INTRODUCCIÓN}

En el último decenio se ha producido un aumento explosivo de nuevas universidades con un aumento exponencial en la oferta de matrícula. El año 1999 de una oferta total de 400.000 matrículas, el 50\% aproximadamente correspondía a universidades nuevas, diez años después, el año 2009, se duplicó esta oferta en más de 800.000 matrículas, de las cuáles casi un 33\% (500.000) corresponden a universidades de reciente creación o acreditación (1). Actualmente, en Chile, la formación profesional de enfermeras está siendo afectada por numerosas variables que de una u otra forma afectan la calidad de sus procesos formativos.

La oferta académica es muy diversa y heterogénea. La carrera de Enfermería no ha estado ajena a este proceso, es así como la oferta académica para el año 2011 fue de 122 carreras de enfermería dictadas por 37 universidades, las que poseen entre una y 15 sedes a lo largo del país (1).

Esta masificación en la educación superior trae como consecuencia la necesidad de modificar los métodos tradicionales de los planes de estudios, toma relevancia establecer la forma de atender a numerosos estudiantes, el aspecto vocacional y la amplia gama de habilidades que poseen los nuevos estudiantes. Junto a ello se deben desarrollar nuevos procesos de aseguramiento de la calidad que aborden estos aspectos (2). Crucial se vuelven los procesos de apoyo al aprendizaje en el caso de las carreras de la salud, se trata de un aprendizaje clínico con participación masiva de estudiantes. Esta situación también obliga modificar los sistemas de información pública para que entreguen información oficial atractiva tanto a postulantes de la educación secundaria como del mundo laboral (3).

El problema no es la diversidad de carreras, sino más bien su heterogeneidad con respecto a la implementación y desarrollo de los requisitos mínimos para asegurar una buena calidad de formación de sus estudiantes (4). Estos requisitos deben considerar al menos cuatro aspectos (5):

1. Capacidad de desarrollar un proyecto educativo con pertinencia social, calidad curricular y buen desempeño profesional.

2. La calidad de sus académicos.

3. Contar con condiciones de operación, un sistema de gobierno y administración; y todos los recursos (humanos, materiales, infraestructura y equipos) requeridos para que la carrera pueda dar cumplimiento a las metas y objetivos declarados.

4. Contar con un sistema de control interno para vigilar el cumplimiento de las medidas y decisiones que se toman para explotar las oportunidades y fortalezas institucionales, corregir las debilidades, superar las limitaciones y alcanzar los objetivos propuestos.

Contar con campos clínicos pertinentes constituye una exigencia para el desarrollo de un proyecto educativo. Existen variados estudios (6-9) que dan cuenta de los beneficios y dificultades para establecer una adecuada relación docente asistencial, sin embargo hay pocas experiencias evaluadas que se constituyan en un modelo a replicar.

El propósito de este trabajo es evaluar el impacto en la formación y desarrollo profesionales, del modelo de integración docente asistencial desarrollado entre la Escuela de Enfermería de la Pontificia Universidad Católica de Chile y los Servicios de Enfermería de la Red de Salud UC (CIDAE-UC).

\section{MARCO REFERENCIAL}

La integración docente asistencial (IDA) es definida por la Organización Panamericana de la Salud (OPS) como la "unión de esfuerzos, en un proceso de creciente articulación, 
entre instituciones de servicios de salud y de educación para contribuir a mejorar las condiciones de vida de la colectividad, mediante la prestación de servicios adecuados a las necesidades reales de la población, la producción de conocimientos y la formación de recursos humanos necesarios en un determinado contexto de la práctica de servicios de salud y de enseñanza". Es una búsqueda de mecanismos de coordinación entre la formación del personal de salud y las necesidades de los servicios que, a su vez, respondan a las necesidades de la población $(10,11)$.

En una investigación cualitativa que tuvo como objetivo describir cómo los educadores perciben la integración entre la docencia y los servicios de salud, y la contribución de esta asociación para la ejecución de la Vigilancia de la Salud (SA) en São Paulo, Brasil, reveló que existe una integración entre la docencia y los servicios, principalmente por iniciativa de la academia. Sin embargo la contribución de la comunidad académica para la aplicación de la SA en la región fue incipiente y se limita a acciones aisladas, principalmente porque los educadores no lo consideran una tarea académica (12). Esto se produce porque la universidad contrata a profesionales de la salud que ejercen en los servicios de salud, que tienen un perfil docente, y los incluyen en el programa de pasantías (12). De este estudio surge la importancia de que las instituciones de educación y los servicios de salud valoricen la función docente realizada por los profesionales de salud en el servicio clínico. La consolidación de prácticas innovadoras en escenarios reales requieren de un paso esencial: la transformación del proceso de trabajo y la coherencia entre el proyecto de los servicios y la propuesta educativa en sus dimensiones políticas, técnicas y metodológicas, para que estas iniciativas sean efectivas en la gestión de los servicios y la universidad; y para la salud de la población. Incluye, necesariamente, las negociaciones con el poder político local, especialmente en el contexto de la municipalización de la salud, que se encuentra en curso en Brasil. En esta perspectiva, un trabajo juicioso para establecer prioridades, la construcción de las intervenciones y continuar las acciones son esenciales; y estos procesos requieren de la participación efectiva de los diversos actores (12). El aporte que tienen los campos clínicos en la formación de los profesionales de la salud es considerado por diferentes autores como un pilar fundamental. Se reconocen como el lugar donde logran la integración teóricopráctica, adquieren destrezas, observan modelos de rol, visualizan el trabajo en equipo, apoyan y cuidan a usuarios, familias y comunidades en su recuperación física y psíquica (6).

Algunos autores $(8,9)$ plantean que la práctica, la disciplina y la investigación están íntimamente relacionadas; se entiende que el conocimiento de la disciplina proviene de la relación recíproca entre estas tres áreas, y se constituye en lo que permite a la profesión enfrentar de mejor manera su rol profesional y el ejercicio de su responsabilidad social.

Agramonte et al. (13) en su propuesta de una guía metodológica para la formación de valores en los estudiantes de Enfermería, sostienen que es necesario involucrar a todos los trabajadores del centro de salud y al personal de los servicios donde desempeñan sus labores prácticas los alumnos en este ambicioso y exigente proceso. Cuando se realice el intercambio con este personal, debe hacerse énfasis en que todos los involucrados conozcan la importancia de su intervención en este proceso y lo que se espera con ello.

La relación docente asistencial en Chile ha sido construida como espacio de colaboración entre organizaciones de salud públicas y centros de formación profesional (14).

Esta relación se desarrolla principalmente a partir de la formación de profesionales en hospitales de mayor complejidad y con un marcado énfasis en el diagnóstico y tratamiento de las enfermedades. Esto, de alguna manera, determinó una formación de profesionales de la salud con un fuerte compromi- 
so con el servicio público (14).

Actualmente, la necesidad de formación de recursos humanos en salud en cantidad y calidad adecuada a los requerimientos del sistema de salud impulsan el desarrollo de una relación docente asistencial que fortalezca una contribución social de quienes la integran, a través de elementos como: a) Desarrollo de un clima de confianza reciproca, b) compromiso institucional de largo plazo, c) extensión del aporte a la salud de la población a través de una contribución al desarrollo armónico de las instituciones integrantes, el servicio y la universidad, d) considera a los servicios de salud y sus entidades como centros que, además de su función asistencial, poseen una función formadora y e) les otorga a la universidades y centros de formación técnica un rol pedagógico importante, que va más allá de la enfermedad y su tratamiento e incluso del área de salud propiamente tal, alcanzando otras disciplinas como gestión del sistema de salud y redes asistenciales y áreas no hospitalarias como son los centros de salud ambulatorios y el trabajo comunitario. Los define como "Campos de Formación", más que "Campos Clínicos", para marcar la diferencia entre un rol histórico restringido a uno más moderno y amplio (14).

La integración de la docencia, la asistencia y la investigación también es un elemento fundamental para los centros formadores $y$ los campos de formación, ya que es el camino hacia una relación docente asistencial saludable, que permita avanzar hacia una mayor contribución al progreso del país.

Otro aspecto que afecta la IDA y que se presenta en países latinoamericanos, como Perú y también Chile, es la sobrepoblación de estudiantes en los campos clínicos producto de un incremento en el número de facultades y carreras de salud que incrementa la demanda por campos clínicos. Esta sobrepoblación genera situaciones que deben ser tomadas en cuenta, como el deseo del paciente de ser evaluado por alumnos, la cantidad de alumnos que pueden examinarlos, entre otras, lo que ha ido generando regulaciones y normativas para evitar una saturación de alumnos en práctica o incomodidad hacia el paciente (15).

Los centros de formación de los profesionales de la salud, también llamados campos clínicos, se ubican en los servicios de salud, situación que es de antigua data y tradicional en la mayoría de los países de Latinoamérica en que los procesos de aprendizaje de los médicos y otros profesionales de la salud se han concentrado en los grandes centros asistenciales (15). Esta situación no siempre garantizó ambientes de formación idóneos para el desarrollo de competencias específicas que necesariamente se deben aprender en los sitios en donde se prestan servicios asistenciales. Las prácticas docentes con frecuencia eran improvisadas y cambiantes. Una de las causas de esta situación radica en la escasa preparación del proceso. Las instituciones educativas y las asistenciales suelen realizar contactos preliminares para establecer un acuerdo para la docencia en el servicio, pero posteriormente la responsabilidad del proceso formativo en servicio queda casi exclusivamente en las instituciones prestadoras de servicios. Es decir, desde un principio no existe la responsabilidad compartida. Como consecuencia, los objetivos educativos expresados no mantienen relación con la práctica cotidiana: los procesos se organizan y se ejecutan de acuerdo con la organización y funcionamiento de la institución asistencial sin que haya una planificación adecuada que tenga en cuenta las necesidades de la docencia. Este problema se puede apreciar en cualquier tipo de entrenamiento práctico como las rotaciones del pregrado, el internado e incluso las residencias clínicas o los programas de postgrado en general (16).

La relación actual se fundamenta en un vínculo contractual de conveniencias y beneficios en la que cada parte busca obtener las mejores condiciones (16).

Esta situación ha significado la revisión 
de las relaciones, comunicación y formas de trabajo entre docencia y asistencia, el establecimiento de requisitos que protejan la seguridad asistencial y el respeto por el paciente, el análisis de las necesidades de desarrollo que tiene el campo clínico y la fortalezas que tiene el centro formador para aportar a este desarrollo; lo que se puede considerar un progreso para una integración beneficiosa. Sin embargo, también ha significado un aumento en la gestión académica por los procesos de planificación conjunta, necesidad de desarrollar nuevos sistemas de registros e información académica y procedimientos de inserción de los estudiantes en los hospitales y servicios clínicos. También se observa un aumento en los requerimientos de recursos académicos e infraestructura docente en los campos clínicos y finalmente la dificultad por lograr acuerdos en cuanto a indicadores como el número de estudiantes por pacientes en cada servicio clínico, la cantidad de docentes y tutores clínicos requeridos.

Todos estos son elementos fundamentales para la articulación e implementación de una docencia integrada en los servicios clínicos, que permita beneficios y logros mutuos. Es aquí donde surge la acreditación que pretende promover la formación del personal de salud altamente calificado, tanto para el ejercicio de su profesión, como para las actividades académicas de docencia e investigación. Busca además proporcionar información confiable a la sociedad sobre las instituciones y sus programas para ampliar así su capacidad de elección. Toda institución que preste servicios debe introducir mecanismos no sólo para garantizar su calidad, sino para brindarles a sus usuarios claridad para facilitar la toma de decisiones. En este contexto, en Colombia se desarrollaron recomendaciones de estándares de acreditación para centros de formación y para escenarios de práctica (16). Se observa que generalmente las instituciones educativas, en su afán por conseguir servicios clínicos para las prácticas de sus estudiantes, no ha- cen un análisis previo sobre los antecedentes de competencias y calidades, y sobre la idoneidad de la institución con la que van a celebrar el convenio. No hay una habilitación previa (entendida como la exigencia de cumplimiento de requisitos esenciales) por parte de los entes gubernamentales competentes que garantice que se cumplan parámetros mínimos de calidad en las instituciones con las que establecen convenios docente-asistenciales (16).

Los escenario de prácticas en salud son una estructura organizativa, producto de la alianza entre una institución formadora y una institución de servicios, con el objetivo específico de llevar a cabo procesos educativos, mediante la integración de la docencia y el servicio, para el desarrollo de competencias teórico-prácticas en salud. Los estándares básicos propuestos para este nivel son: procesos educativos, recursos humanos, instalaciones, y dotaciones y recursos educativos (16).

Los centros de formación en salud son definidos como un conjunto de escenarios de docencia servicio en cuya misión y objetivos, y por ende en su organización y funcionamiento, se hace énfasis en actividades académicas centradas alrededor de la prestación de un servicio en salud. Los estándares básicos de calidad para los centros de formación en salud propuestos incluyen tres factores: proyecto institucional, recursos humanos, e interacción con los usuarios y la población (16).

El modelo de Integración Docente Asistencial (CIDAE-UC) que se presenta en este trabajo corresponde a la experiencia sistemática de colaboración mutua desarrollada entre la Escuela de Enfermería de la Pontificia Universidad Católica de Chile (EEUC) y los Servicios de Enfermería que conforman la Red de Salud UC.

La Red de Salud UC es un campo formador constituido por un conjunto de establecimientos asistenciales de salud (campos clínicos), con distinto grado de complejidad 
y condiciones de estructura, personal y equipamiento tecnológico adecuado para realizar la formación de profesionales y técnicos para la salud.

Cabe señalar que un hospital o clínica adquiere el carácter de "docente" cuando consigue mantener, de forma prolongada, un ambiente apropiado para el proceso de enseñanza-aprendizaje. Con este propósito es importante considerar la vocación docente del hospital tanto en la descripción de su misión como en las políticas de la organización, privilegios y obligaciones de los profesionales relacionados con la institución. Junto con esto, es importante poner especial atención en que la estructura, organización, modelo de gestión y características de los docentes, sean adecuadas y ejemplares para la formación de los alumnos (17).

La Red de Salud esta constituida por las siguientes instituciones: Hospital Clínico UC, Clínica UC, Clínica San Carlos UC, Centro Médico San Joaquín, Unidades de Toma de Muestras y el Centro de Cáncer Nuestra Señora de la Esperanza.

La IDA de Enfermería UC es entendida como un sistema de cooperación mutua entre enfermeras de asistenciales de la Red de Salud UC y profesoras de la Escuela de Enfermería UC, que tiene como meta integrar a enfermeras docentes y clínicas, mediante actividades y procesos de interés mutuo y crear las condiciones para que se desarrolle una sinergia que beneficie la docencia, asistencia, investigación y extensión y permita desarrollar modelos de atención innovadores y coherentes con la formación profesional y la excelencia clínica.

Para la formación de los profesionales en salud es incuestionable la necesidad de desarrollar diferentes experiencias de atención directa con el paciente para adquirir habilidades clínicas; las que por lo general, se dan en los cursos clínicos e internado. Actualmente la tendencia es a la exposición temprana a los pacientes en los primeros años, para mejorar la comprensión y rendimiento de los estudiantes en los cursos preclínicos (18).

La importancia de la sincronización de experiencias en enfermería y una colaboración estrecha entre enfermeras docentes, estudiantes y enfermeras clínicas, dará a los estudiantes un balance apropiado de perspectiva, habilidades clínicas y académicas, que lo prepararán efectivamente para satisfacer las necesidades de cuidados de salud de los pacientes, ya que de lo contrario, el conocimiento teórico que adquieren los estudiantes de enfermería, está en riesgo de separarse por completo del conocimiento clínico.

En base a estos antecedentes, surge la urgencia de buscar modelos de integración docente-asistencial que sean eficientes, eficaces y costo efectivos, con ventajas mutuas para la calidad de la atención y para el mejoramiento de la formación profesional de enfermeras. Lo cual no es una tarea fácil, ya que en la práctica se observa con frecuencia que las enfermeras clínicas visualizan a los alumnos como una carga adicional a sus responsabilidades cotidianas, que la rotación de enfermeras interfiere en el proceso de modelaje ya que las decisiones de cambios de enfermeras se basan exclusivamente en las necesidades asistenciales y que no está considerado el tiempo ocupado en docencia por las enfermeras asistenciales.

La implementación de un modelo de integración, requiere entonces, no solamente de un trabajo consensuado entre enfermeras docentes y asistenciales, sino también de una voluntad política de las autoridades que permita contar con las condiciones que favorezcan la integración docente-asistencial.

En un estudio exploratorio realizado el 2008 en la ciudad de Concepción, Chile, se identificaron dos obstáculos primordiales para que exista una verdadera IDA, ellos son: 1) La falta de estímulos, ya que la academia no reconoce en los asistenciales su participación en la docencia y 2) La nula o escasa 
capacitación que se les otorga por parte de la universidad. Las sugerencias para optimizar la Integración Docente Asistencial entre la academia y la asistencia se relacionan con mejorar la comunicación y motivar a las enfermeras clínicas a que participen en la docencia mediante el reconocimiento de la academia hacia ellas. Un punto fundamental que mencionan es la experiencia de las docentes en la práctica clínica, para así poder integrarse en el campo clínico (19).

El modelo de integración desarrollado consiste en la creación de una unidad funcional formal de trabajo sistemático, el Comité de Integración Docente Asistencial de Enfermería de la Universidad Católica (CIDAE-UC), que es el organismo encargado de facilitar, promover y articular acciones que favorezcan el aprendizaje e incremento de habilidades del alumno de enfermería y que propicien el desarrollo de las enfermeras asistenciales en un nivel de excelencia, que beneficie a la asistencia y a la docencia.

De este modo, el CIDAE-UC queda conformado por:

- La coordinadora docente asistencial, profesora de categoría adjunta de la Escuela de Enfermería, quien lo preside.

- Dos académicos de la Escuela de Enfermería.

- Cuatro jefes de Enfermería de los hospitales y clínicas de la Red de Salud UC.

- Cuatro enfermeras coordinadoras de los centros ambulatorios y unidades de toma de muestras de la Red de Salud UC.

La coordinadora docente asistencial asume la responsabilidad de cumplir con los siguientes objetivos estratégicos:

- Desarrollar un modelo de Integración Docente Asistencial (IDA) que favorezca y posibilite el aprendizaje del alumno de enfermería, en forma integral y coherente en toda la Red de Salud UC.
- Crear instancias de encuentro y trabajo conjunto entre enfermeras de la Red UC y profesoras de Escuela de Enfermería, para el análisis y desarrollo de la actividad asistencial, docente, de investigación y extensión.

- Propiciar el desarrollo de los profesionales de enfermería UC, en un nivel de excelencia que responda a las necesidades de salud de los usuarios de la Red de Salud UC.

En torno a estos objetivos se desarrolla el Modelo de Integración Docente-Asistencial (CIDAE-UC) como producto de un trabajo sistemático y continuó que se inicia el año 2000. En el esquema 1 se pueden reconocer las cinco etapas con sus principales hitos que son fundamentales para su desarrollo:

- Etapa 1: Diagnóstico de la situación: que se realizó mediante una encuesta sobre expectativas de integración y desarrollo a 336 enfermeras de la Red de Salud UC y 7 académicos de la EEUC. Las principales expectativas y beneficios de la IDA percibidos por enfermeras clínicas y docentes EEUC: $1^{\circ}$ Proyección conjunta con un $37 \%, 2^{\circ}$ actualización y capacitación con un $29 \%$ y $3^{\circ}$ Aumentar y compartir conocimientos con un $27 \%$.

- Etapa 2: Desarrollo de líneas estratégicas para el fortalecimiento de la IDA, dirigidas a cumplir con expectativas de Red UC y Escuela de Enfermería UC (EEUC).

- Etapa 3: Implementación de una estructura permanente para favorecer la IDA en enfermería denominada: Comisión de Integración Docente Asistencial (CIDAE UC).

- Etapa 4: Organización de un sistema de comunicación e información interna entre la Red de Salud UC y EEUC.

- Etapa 5: Evaluación anual de los logros de CIDAE UC y reformulación de sus actividades. 
Esquema 1. Línea de Tiempo con las etapas y principales hitos de Desarrollo del Modelo de Integración Docente Asistencial de Enfermería (CIDAE-UC). 2000 - 2010.

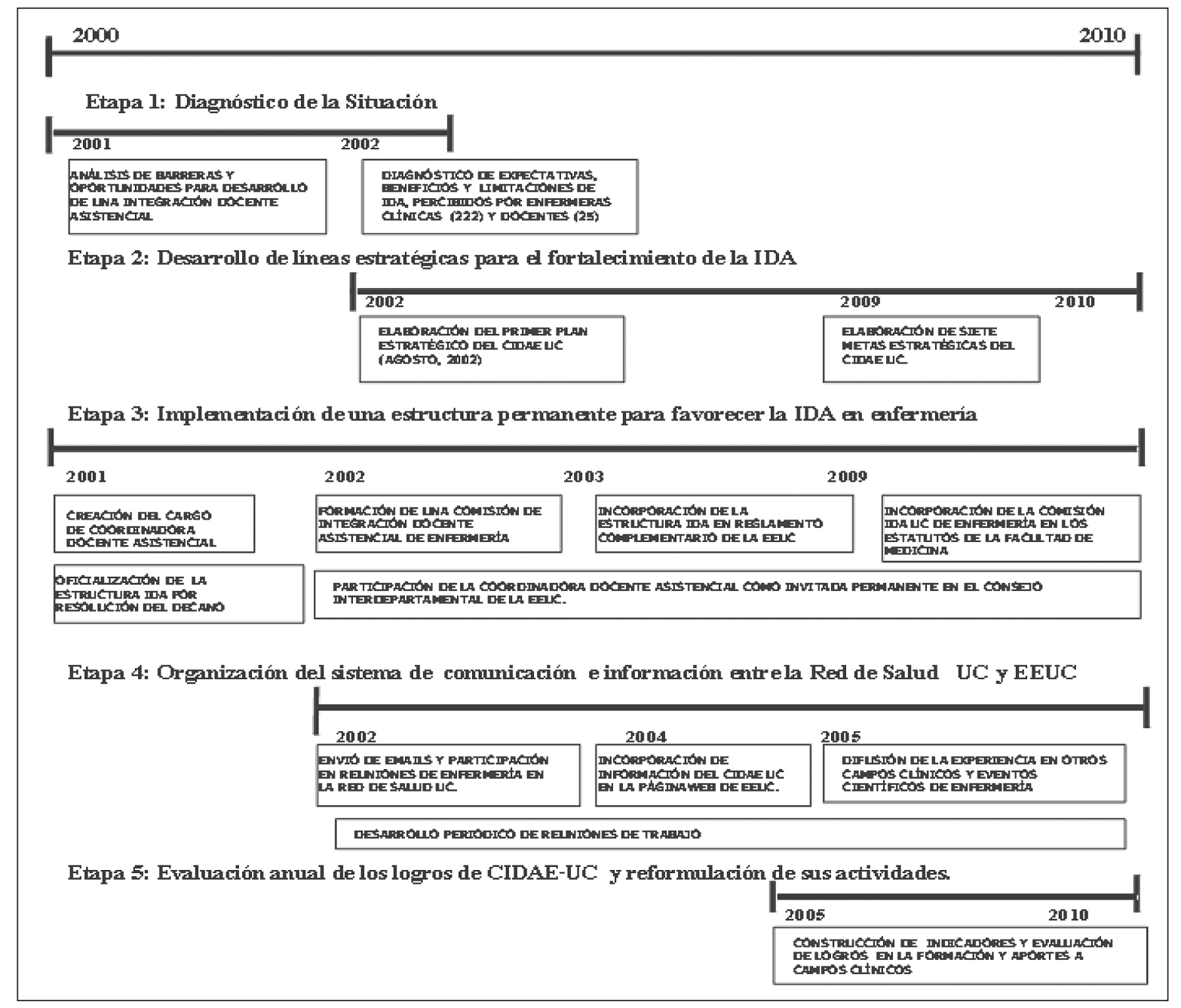

\section{MATERIAL Y MÉTODO}

Estudio descriptivo, de corte transversal, que analiza los datos de las memorias del Comité de Integración Docente Asistencial de Enfermería de la Universidad Católica (CIDAEUC) de los años 2000 - 2005 y 2009.

Las variables estudiadas son:

Aportes del modelo en la formación profesional

- Optimización del sistema de programa- ción y planificación de la docencia.

- Pertinencia para la formación de las áreas clínicas de la Red de Salud UC.

- Incremento de docencia en áreas clínicas de la Red de Salud UC.

Efecto del modelo en el desarrollo profesional de las enfermeras clínicas

- Incremento de enfermeras clínicas que apoyan la docencia.

- Incorporación de enfermeras clínicas a la planta académica.

- Oportunidades de perfeccionamiento profesional. 
- Fomento de actividades colaborativas de extensión e investigación.

El análisis de datos fue cuantitativo-descriptivo mediante medidas de tendencia central, gráficos y tablas.

Con relación a los aspectos éticos, cabe señalar que este estudio utiliza información pública de las memorias del trabajo realizado por el CIDAE-UC, y serán citados anónimamente, con lo cual se resguarda la confidencialidad.

\section{RESULTADOS}

\section{Aportes del modelo en la formación pro- fesional}

Optimización del sistema de programación y planificación de la docencia

La coordinadora docente asistencial junto a las coordinadoras de servicios y a las jefaturas de enfermería de los centros asistenciales, reclutan a las enfermeras que asumirán la guía de cada alumna, según competencias y disponibilidades. Con este trabajo coordinado y planificado se logra desarrollar un registro de las enfermeras que participan activamente en docencia clínica, con datos del centro y servicio donde trabajan, la trayectoria en la institución, los cursos de pregrado en que participan y los programas de formación que han realizado. Este registro ha permitido:

- Mantener un seguimiento de la participación en docencia clínica y posibilita un mayor acercamiento para brindarles oportunidades de desarrollo profesional, que redunda en una mejor atención al usuario y la posibilidad de constituirse en buenos modelos para los futuros profesionales.

- Entregar un reconocimiento de la EEUC, en forma anual y sistemática, a través de una carta por su participación en docencia de estas enfermeras clínicas.

- Propiciar oportunidades de desarrollo profesional a través de cupos y becas para los distintos programas de educación continua, especialización y postgrado que ofrece la EEUC.

- Identificar a las enfermeras/os que, por su aporte meritorio y compromiso, son merecedoras de un nombramiento académico. Este nombramiento consiste en su incorporación a la planta académica en categoría académica especial, denominada Categoría Académica Adjunta. La CIDAE-UC desarrolla e implementa estrategias para motivar y apoyar a estas enfermeras para su postulación a nombramiento académico en la EEUC. Una de estas estrategias es establecer, a modo de referencia, ciertas competencias e indicadores de docencia clínica para la orientación de las enfermeras clínicas en su postulación y para guiar la categorización académica.

- Contar con un sistema de información mínima y necesaria para efecto de la acreditación de los campos clínicos: a) Un "Registro Académico" que contiene toda la información individual o grupal de los alumnos en experiencia clínica (Programas de cursos, objetivos prácticas, perfiles de competencias y docentes a cargo) y b) Procedimiento para cumplir con el requisito de inducción y registro académico para alumnos de enfermería en prácticas realizadas en campos clínicos de la Red de Salud UC.

Pertinencia para la formación de las áreas clínicas de la Red de Salud UC

El Gráfico 1 muestra que los estudiantes que participan en sus experiencias clínicas se distribuyen entre un $45,7 \%$ en la Red de Salud UC y un $54,3 \%$ en otros centros formadores. Los campos clínicos que no son de la Red de Salud UC corresponden principalmente a 
Grafico 1: Distribución de los estudiantes según campo clínico año 2009.

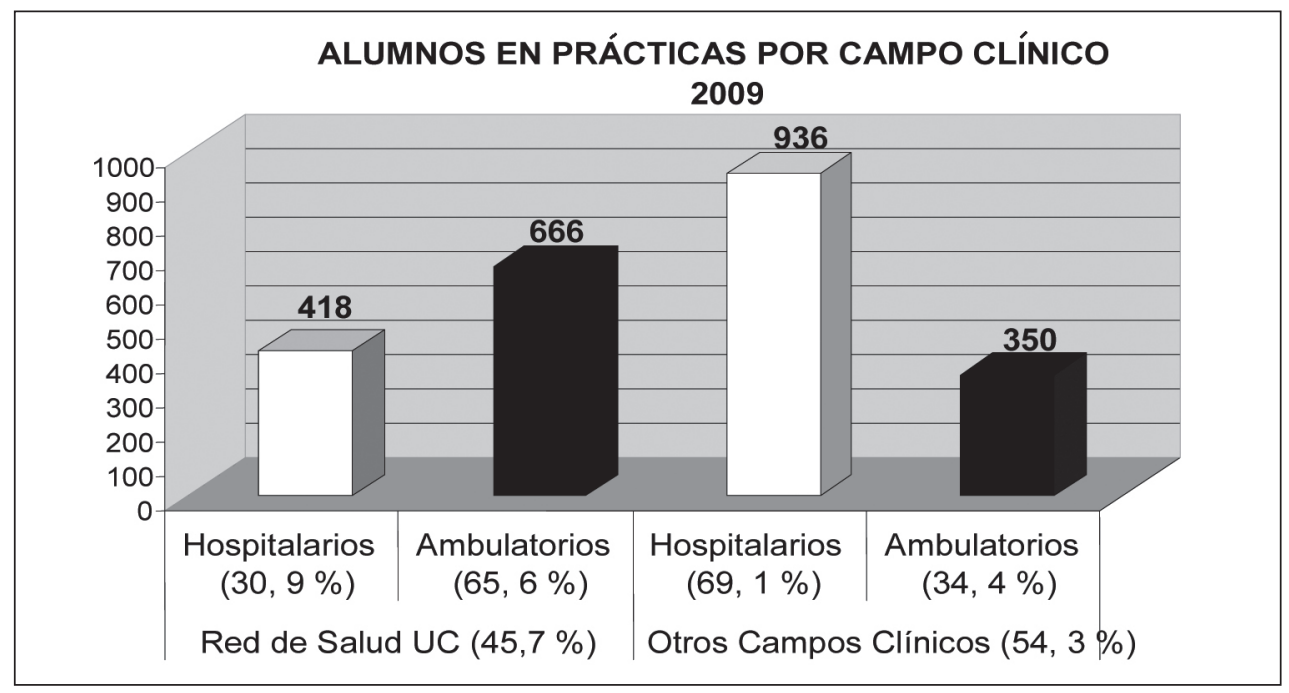

instituciones públicas de salud, hospitales y centros de salud familiar.

Esta situación muestra la pertinencia de la distribución de los campos clínicos utilizados para la formación profesional. Es necesario que el alumno tenga experiencias de cuidado de enfermería en usuarios de menores ingresos que acceden al sistema público de salud, que en Chile corresponde al 76,9\% de la población (20).

Incremento de docencia en áreas clínicas de la Red de Salud UC

El Gráfico 2 muestra un importante incremento de la docencia en áreas clínicas de la

Gráfico 2: Tendencia en la cantidad de experiencias clínicas realizadas en la Red de Salud.

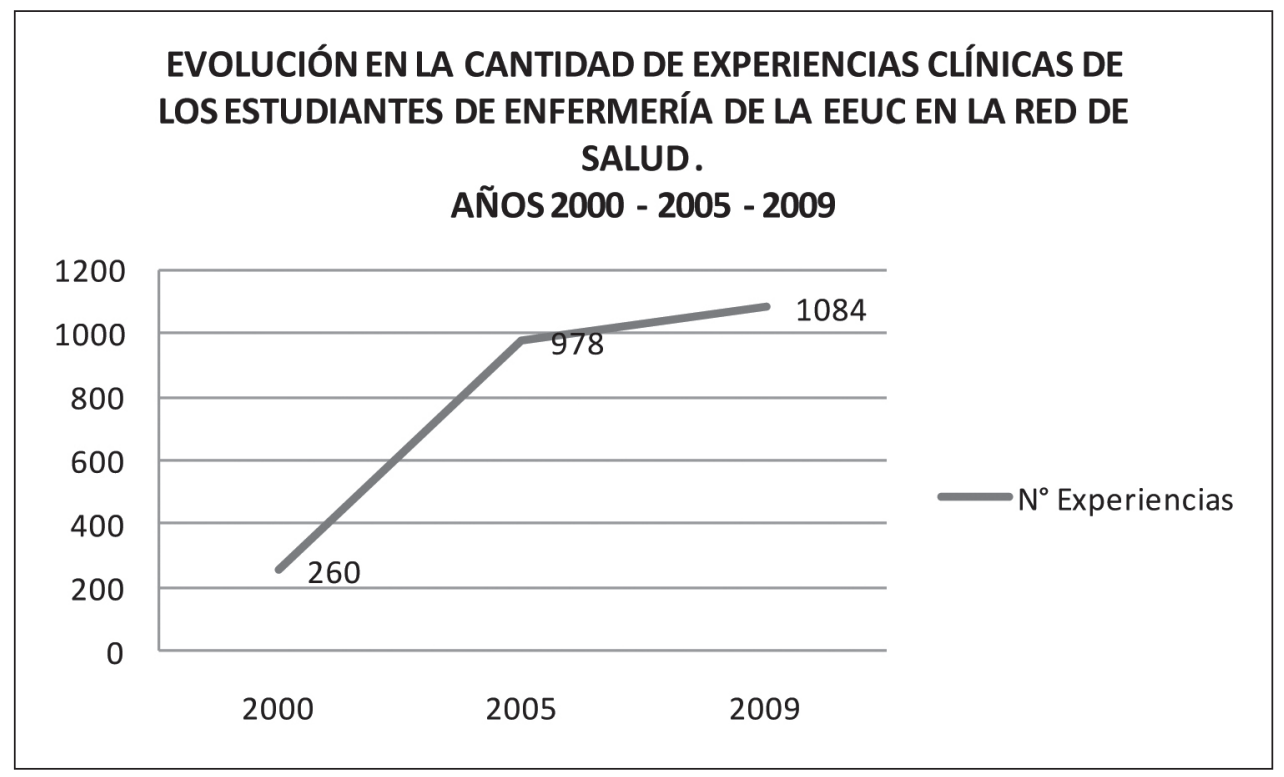


Red de Salud UC. La cantidad de experiencias clínicas en el último decenio aumentó en un $75 \%$ (se cuadriplicó).

Las experiencias clínicas de los alumnos de enfermería del Programa de Pregrado que requieren mayor participación de las enfermeras clínicas en su formación, son: la Práctica Autogestionada (PAG) realizada entre el $2^{\circ}$ y $3^{\text {er }}$ año de la carrera, los Internados realizados entre el $4^{\circ}$ y $5^{\circ}$ año y los cursos de profundización del Tramo Profesional de En- fermera y de Enfermera Matrona realizados en el $5^{\circ}$ año. Las enfermeras clínicas asumen la responsabilidad de monitorear, supervisar y evaluar la práctica del alumno junto con el profesor escuela a cargo.

El Gráfico 3 muestra que la evolución del número de alumnos que realiza práctica autogestionada (PAG) presenta un 'peak' el año 2005 para luego presentar una disminución el año 2009, sin embargo la tendencia central del período es ascendente.

Gráfico 3: Evolución del número de alumnos que realiza práctica autogestionada en la Red de Salud UC. Años 2001 - 2009.

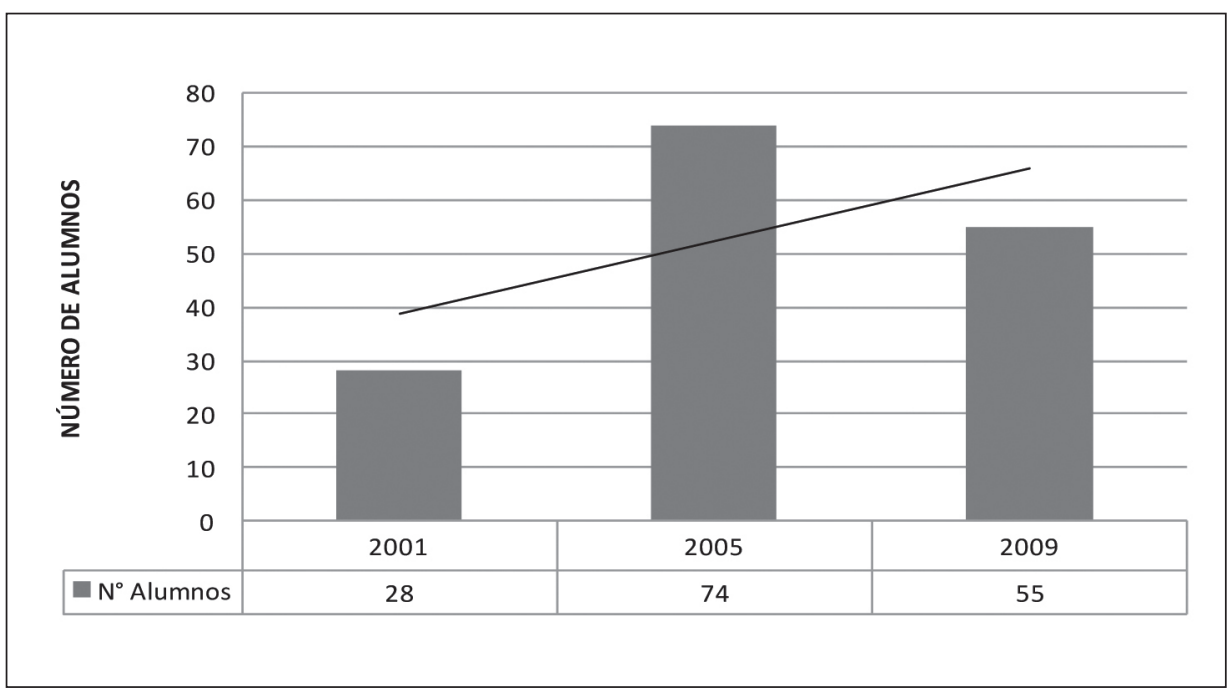

La observación de cinco años de experiencia muestra que sobre el 70\% de los alumnos eligen los campos clínicos de la Red de Salud para efectuarla, realizándola un número estable de alumnos durante los períodos 2006 a 2008, disminuyendo el número de alumnos el año 2009 porque se abre la posibilidad que un mayor número de alumnos realice la PAG en el Hospital Sótero del Río.

El Gráfico 4 muestra el incremento en un $60 \%$ del número de alumnas que realiza su internado en los campos clínicos de la Red de Salud UC.

Las prácticas clínicas de internado que se registran durante el año 2009 son 187, distribuidos en Internados Hospitalarios del Adulto y del Niño, Internados Hospitalarios y Ambulatorios de Enfermera Matrona e Internado Ambulatorio de Enfermería del Niño y Adulto. Se realizan en períodos de 14 semanas, con el $45,9 \%$ en campos clínicos de la Red de Salud UC y el 54,1\% en otros campos clínicos. 
Gráfico 4: Evolución en la cantidad de internas de enfermería en la Red de Salud UC. Años 2001, 2004 y 2009.

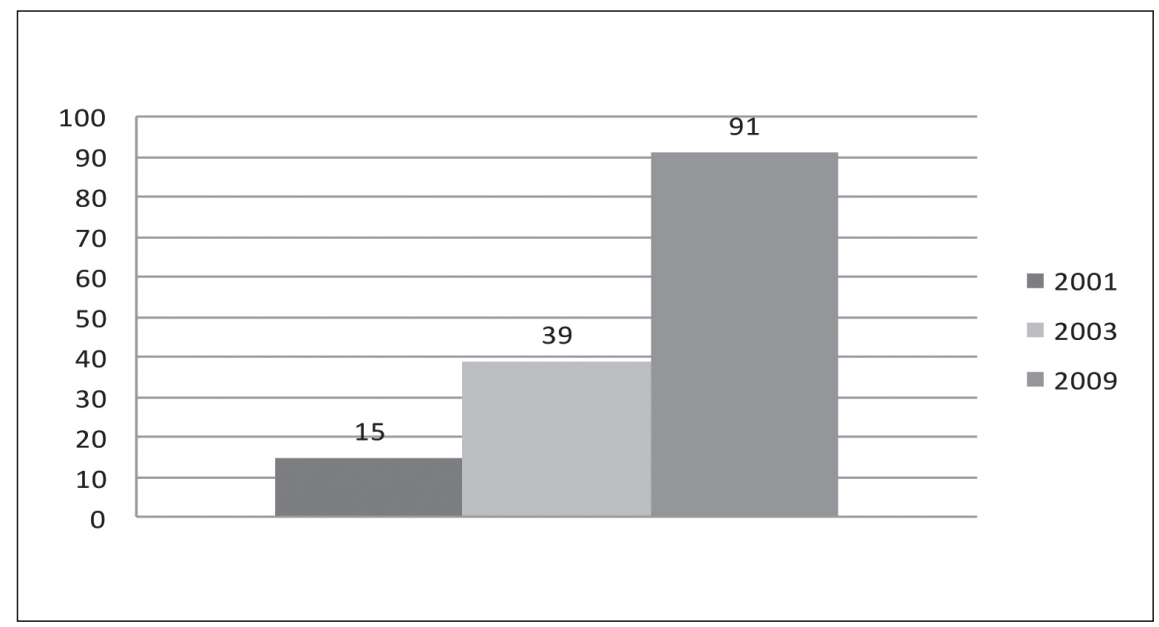

II. Efecto del modelo en el desarrollo profesional de las enfermeras clínicas

Incremento de enfermeras clínicas que apoyan la docencia
El Gráfico 5 muestra el importante incremento en la cantidad de enfermeras clínicas de la Red de Salud UC que asumen la responsabilidad de ser tutores de los alumnos de Pregrado. De 25 el año 2001 aumentan a 149 el año 2009.

Gráfico 5: Evolución en la cantidad de Enfermeras/os Clínicas/os de la Red de Salud UC que apoyan las experiencias de los alumnos de Pregrado de la EEUC. Años 2001, 2004 y 2009.

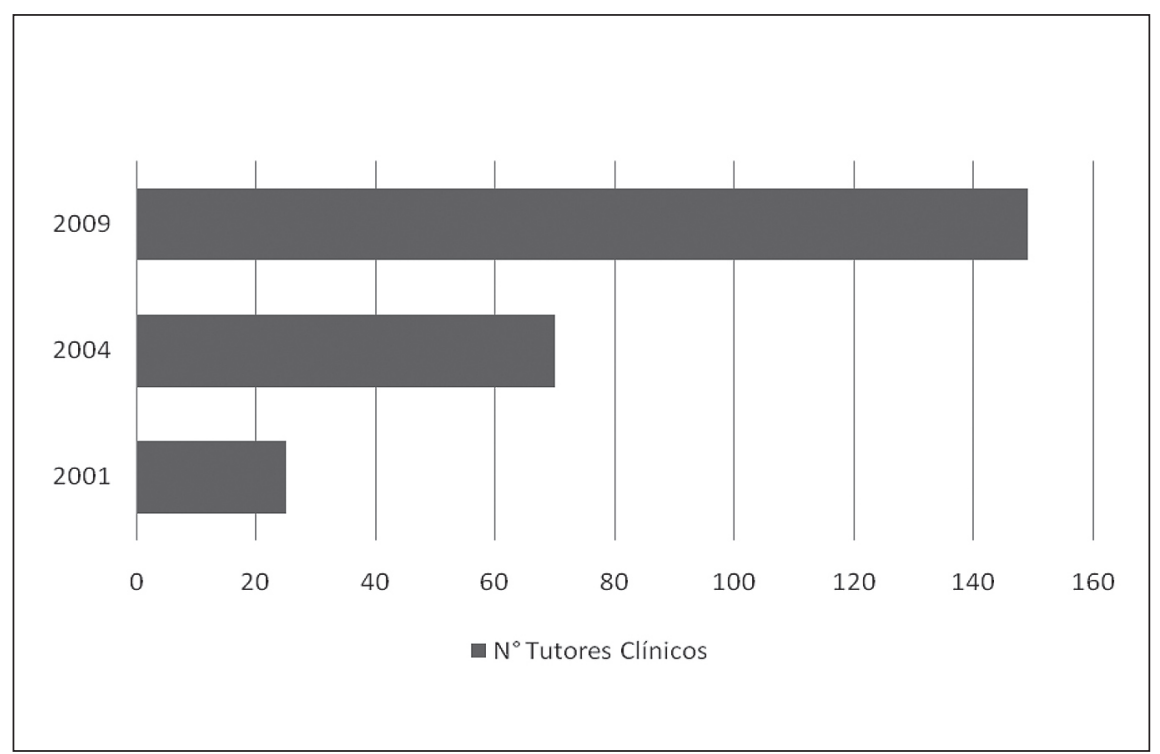


Incorporación de enfermeras clínicas a la planta académica

Se observa en el Gráfico 6 un aumento en la cantidad de nombramientos académicos de enfermeros/as clínicos/as de la Red de
Salud UC, duplicándose entre los períodos 2001 - 2003 con un promedio de 14,5 y en el período 2005 - 2009 con un promedio de 29 nombramientos. La categoría académica predominante en los nombramientos es la de Instructor Adjunto.

Gráfico 6: Evolución en la cantidad de nombramientos académicos de enfermeros/as clínicos/as de la Red de Salud UC. Años 2001 al 2009.

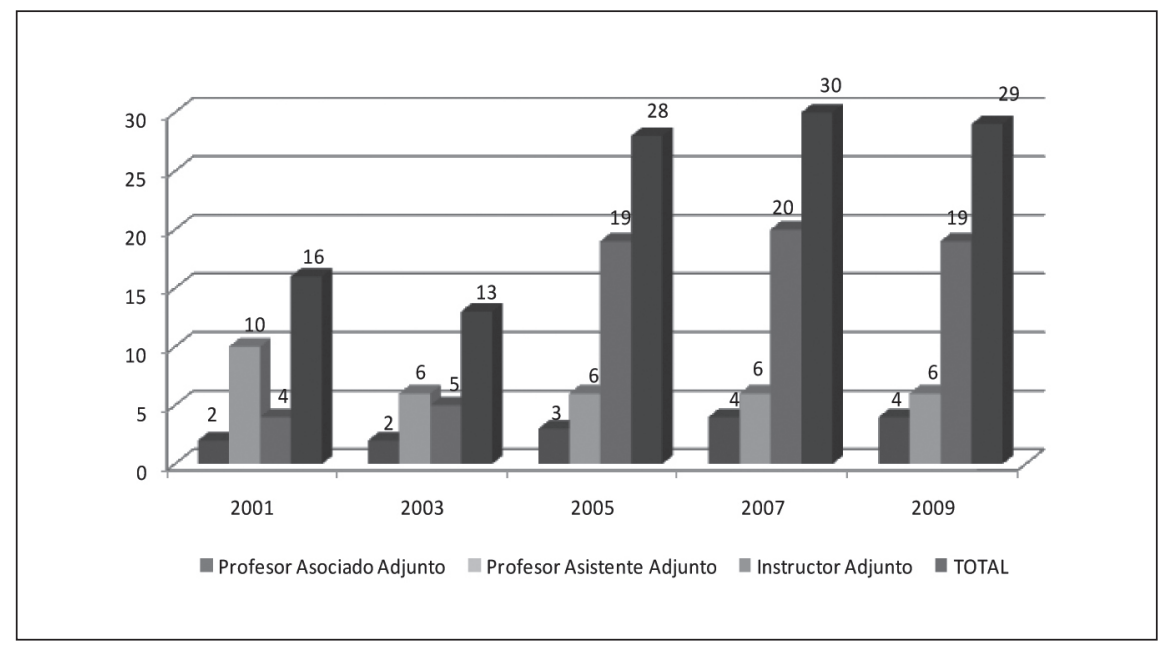

Oportunidades de perfeccionamiento profesional

Las enfermeras clínicas de la Red de salud
UC tienen la oportunidad de acceder a becas para estudiar en los diferentes programas que ofrece la EEUC. El Gráfico 7 muestra el importante aumento en la cantidad de en-

Gráfico 7: Evolución del perfeccionamiento de enfermeras/os clínicas/os de la Red de Salud UC en programas académicos de la EEUC. Años 2002, 2005 y 2009.

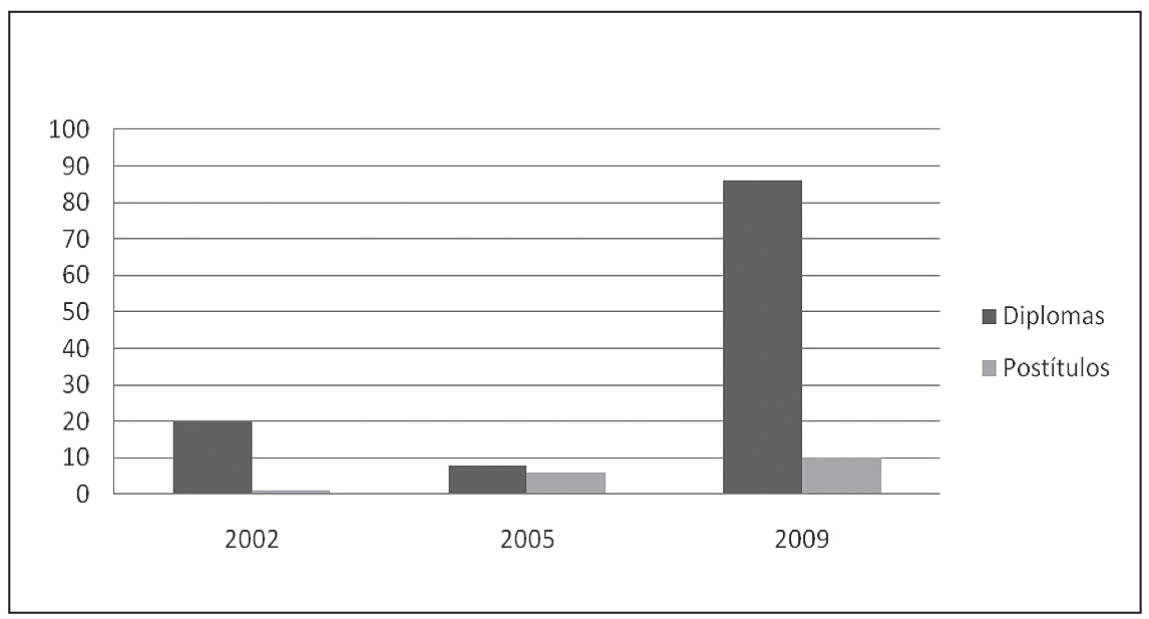


fermeras/os clínicas/os de la Red de Salud UC que realizan perfeccionamiento en programas académicos de la EEUC. Llama la atención el marcado aumento en la realización de diplomados, de 20 el año 2002 a 80 el año 2009, versus los programas de postítulos que aumentan entre los años 2000 y 2009 de 2 a 10.

Al revisar los programas de formación profesional que ofrece la Escuela de Enfermería y confrontarlas con cada profesional que asume la responsabilidad de docencia clínica en alumnos de pregrado durante el año 2009 , se observa que el $34 \%$ de los docentes clínicos del hospital han realizado programas de formación y más de la mitad (57 $\%$ ) de los docentes clínicos de los Centros de Salud Familiar ANCORA UC. El Diplomado de Tendencias Innovadoras de Docencia en Enfermería es el programa más cursado por las enfermeras Hospital Clínico UC y los postítulos de Enfermería en los Centros de Salud Familiar ANCORA UC.

Entre los años 2000 y 2009 realizaron postítulos de especialización 66 enfermeras de la Red de Salud UC, siendo el Postítulo Enfermería del Adulto con problemas Cardiacos el más realizado (16 enfermeras), seguido por el Postítulo Enfermería del Adulto con problemas Nefrológicos (12 enfermeras).

Entre los años 2000 y 2001, con el apoyo de la Dirección de Investigación y Postgrado de la Universidad (DIPUC), se desarrollan dos programas docentes asistenciales para la promoción y autocuidado de la salud; uno en el área infantil y otro para adultos mayores.

Estos programas se transformaron en un modelo de demostración para la asistencia, que fortalece el trabajo interdisciplinario al interior de la Facultad de Medicina y se constituye en un polo de interés para enfermeras extranjeras.

Fomento de actividades colaborativas de extensión e investigación

El CIDAE, como modelo de relación y co- municación, permite impulsar proyectos de desarrollo docente asistencial y de investigación generada en la práctica de enfermería.

Entre las actividades colaborativas de extensión cabe destacar la creación, entre los años 2003 y 2007, de siete Diplomados Clínicos en los que se potencia el trabajo conjunto de enfermeras clínicas del Hospital Clínico UC, la Clínica San Carlos y enfermeras docentes de la EEUC.

Dos proyectos, que son liderados por enfermeros clínicos de la Red de Salud UC y que además poseen nombramiento de profesor adjunto en la EEUC, obtuvieron financiamiento externo del Fondo Nacional de Investigación y Desarrollo en Salud (FONIS), los años 2007 y 2010.

\section{DISCUSIÓN Y COMENTARIO}

La importancia que han tenido los campos clínicos en el desarrollo y la educación de la salud en Chile es irrefutable y se debieron a la colaboración recíproca entre las universidades y los hospitales públicos, estableciéndose de este modo la relación docente asistencial. En la actualidad, con el aumento de los estudiantes de las carreras de la salud y los imperativos de calidad y seguridad asistencial, esta relación pasa a ser objeto de regulaciones y está sujeta a convenios. Es un concepto que ha evolucionado más allá de una relación, requiere de integración, coordinación y trabajo planificado.

En este contexto se describe la experiencia de diez años de integración docente asistencial entre la EEUC y la Red de Salud UC, a través de mostrar los principales hitos que permitieron su desarrollo, y los logros obtenidos en términos del incremento de su colaboración en la formación de los futuros profesionales y en el aumento del desarrollo profesional de las enfermeras clínicas mediante capacitación, actividades de extensión y de investigación. 
Es un modelo de trabajo amplio y participativo, que ha resultado positivo en su evaluación, que es posible replicar y mejorar. Sin embargo, no existe en la literatura un modelo similar, lo que impide comparar sus resultados con otros modelos. Se describen y evalúan experiencias más acotadas y específicas que destacan el rol del campo clínico en la formación, se analizan sus beneficios y también las numerosas dificultades existentes.

Una limitación del trabajo presentado es que no se estudió la opinión de las enfermeras clínicas sobre las expectativas de integración y desarrollo postimplementación del modelo CIDAE-UC, lo que habría sido un aporte valioso a la evaluación del impacto logrado.

Las características del modelo descritas y las variables medidas se contrastan con la literatura ya que abordan los principales aspectos descritos por otros autores, como son la importancia del apoyo político de la institución formadora, establecer clima de confianza reciproca, la necesidad de incorporar estrategias de integración entre enfermeras docentes y clínicas en actividades conjuntas como son el desarrollo de actividades de extensión y de investigación, y considerar la necesidades de reconocimiento, capacitación y desarrollo de las enfermeras clínicas. En este punto cabe señalar que la perspectiva del usuario y la comunidad es un enfoque que se menciona en la literatura, que no se describe y evalúa en este modelo. Sería importante ampliar este modelo en este sentido y evaluarlo en términos de su impacto en la calidad de atención percibida por los usuarios y comunidades; y evaluar las actividades académicas centradas alrededor de la prestación de servicios de salud.

En esta línea, también es necesario proponer estándares e indicadores para acreditar los campos clínicos.

Este es un modelo de trabajo que facilita el desarrollo de la docencia de enfermería en los campos clínicos hospitalarios y ambulatorios, pero no asegura que los resultados del proceso formativo sea de calidad, ya que ello depende de muchas otras variables, como son el proyecto educativo en sí, los procesos de selección de estudiantes que ingresan a la carrera, el académico guía o tutor del estudiante y la congruencia de los procesos de enseñanza-aprendizaje con los objetivos a lograr planteados.

El CIDAE-UC, a pesar de sus limitaciones, es una buena aproximación al tema. Se requieren futuros estudios que aborden con mayor amplitud el concepto de campo clínico o espacio de formación, que evalúe en forma diferenciada el impacto en la calidad de atención.

En los aspectos más políticos se requiere complementar la acreditación de los programas de pre y postgrado de Enfermería con una acreditación de los campos clínicos.

Esta evaluación permite afirmar que el modelo de integración docente asistencial descrito es efectivo, porque a 10 años de su aplicación es posible evaluar los aportes del modelo en la formación profesional y describir el efecto en el desarrollo profesional de las enfermeras clínicas.

Como aportes en la formación profesional se observa optimización del sistema de programación y planificación de la docencia, lo que permite desarrollar un sistema de información de las enfermeras clínicas que realizan docencia y registros académicos. Este resultado es muy consistente con lo encontrado en la literatura ya que se destaca como uno de los elementos fundamentales para cumplir con el concepto más amplio de la IDA, que no siempre se observa ya que generalmente hay mucha improvisación, falta de planificación y trabajo coordinado. Asimismo, los resultados obtenidos que se relacionan con el reconocimiento de la participación en docencia y la incorporación de las enfermeras clínicas como académicas de la EEUC son logros muy importantes ya que son aspectos que se mencionan en la literatura como un obstáculo fundamental para que exista una verdadera IDA. 
La creación de un procedimiento de inducción para los estudiantes, no se menciona como un elemento necesario, sin embargo la pertinencia para la formación de las áreas clínicas de la Red de Salud UC y el incremento de docencia clínica son variables señaladas, en la literatura revisada, como recomendables porque mejoran la comprensión y el rendimiento de los estudiantes. En enfermería proporcionan un balance de perspectiva apropiado y preparan efectivamente para el cuidado de la salud. No obstante, se debe asegurar un ambiente idóneo, el que se logra con una relación adecuada de estudiantes, enfermeras clínicas y pacientes.

Este es un modelo de trabajo que además de mejorar la planificación y programación de la docencia, incrementa la comunicación de la unidad académica de los centros formadores de la Red de Salud UC; por ejemplo se analizan las necesidades de cupos para alumnos en experiencias clínicas y se contrastan con las disponibilidades de los servicios clínicos tanto ambulatorios como hospitalarios. Este aspecto es mencionado, en algunos estudios previos, como fundamental para optimizar la relación docente-asistencial.

El efecto en el desarrollo profesional de las enfermeras clínicas se observa claramente a través de un importante incremento de las enfermeras clínicas que apoyan la docencia, una mayor incorporación de enfermeras clínicas a la planta académica. Ambos resultados son interdependientes, el primero es consecuencia del segundo ya que es un reconocimiento que motiva a las enfermeras clínicas a realizar docencia.

Respecto al aumento del perfeccionamiento profesional encontrado, está claramente también identificado como un elemento significativo para el desarrollo de una verdadera IDA. El crecimiento de actividades colaborativas de extensión no es mencionado como un elemento significativo en la IDA.

Respecto a investigación se presenta un postrero desarrollo, luego de 6 años de instaurado el modelo, sin embargo este resul- tado es una consecuencia lógica de un trabajo colaborativo e integrado y la literatura lo menciona también como elemento fundamental para fortalecer la IDA ya que permite avanzar hacia una mayor contribución al progreso del país.

Desde la perspectiva del campo clínico el aumento progresivo de cupos para experiencias, además de favorecer la formación de los alumnos, impulsa la necesidad de mayor desarrollo de competencias en los profesionales de enfermería de la institución, por la búsqueda necesaria de nuevos conocimientos que permitan enfrentar las preguntas e inquietudes de los alumnos.

$\mathrm{Al}$ analizar el modelo descrito y los resultados obtenidos se puede concluir que el CIDAE-UC: $1^{\circ}$ Facilita el aprovechamiento de conocimientos y capacidades de enfermeras docentes y clínicas en la asistencia y la docencia. $2^{\circ}$ Para asegurar la continuidad y el éxito del modelo es indispensable que exista una estructura formal que considere un cargo de dedicación exclusiva y un comité operativo de carácter permanente. Y $3^{\circ}$ Es una estrategia de valor para preparar un personal idóneo en las diversas especialidades e incrementar la disciplina de enfermería, a través del desarrollo profesional y de la investigación colaborativa.

Finalmente, cabe destacar que este Modelo de Integración Docente Asistencial (CIDAE-UC), descrito y evaluado, aporta al conocimiento con elementos que son claves para desarrollar una adecuada integración docente asistencial y puede ser replicado por otros centros formadores de profesionales de la salud.

\section{REFERENCIAS}

1. Oferta académica de pregrado 2011. [Internet]. Ministerio de Educación de Chile SIES.c2011 [citado19enero2011].Disponible en: Ofertahttp://www.mineduc.cl/ 
index3.php?id_contenido $=13037 \&$ id seccion $=3224 \&$ id_portal $=46$

2. Bernasconi A, Rojas F. Informe sobre la educación superior en Chile 1980-2003. Chile . [Internet]. 2004 [citado 20 octubre 2010]. Disponible en: http://www. uvm.cl/corporativas/vra_docs/Informe $\% 20$ educ\%20superior\%20en $\% 20$ Chile.pdf.

3. Raczynsky D, Canales A. Demanda de información sobre las carreras de pregrado en la educación superior chilena. [Internet]. Santiago de Chile: Seminario Internacional Información en la Educación Superior. Necesidades y Propuestas; 2001 [citado 20 junio 2011]. Disponible en: www.cse.cl/ASP/WEB_CSEpublic_ pdf.asp

4. Muga A, Sotomayor A. La diferenciación por calidad, ¿cuáles son los límites? Revista Calidad en la Educación. 2004; (21): 47-60.

5. Colombia Unidades Tecnológicas de Santander. Documento Institucional: Sistema de Autoevaluación y autorregulación de la calidad. 2005 CNA Colombia.

6. Salgado E, Sanhueza O. Enseñanza de la Enfermería y relación docente asistencial en el marco educacional y sanitario chileno. Invest. educ. enferm. 2010; 28(2): 258-266.

7. Lagrosen S, Seyyed-Hashemi R, Leitner M. Examinations of the Dimensions of Quality in Higher Education. Quality Assurance in Education. 2004; 12(2): 6169.

8. Salas P, Ramón S. La calidad en el desarrollo profesional: avances y desafíos. Rev Cubana Educ Med Super. [Internet]. Mayo-ago. 2000 [citado 10 junio 2011]; 14(2): p. 136-147. Disponible en: $<$ http://scielo.sld.cu/scielo.

9. Arteaga HJ, Chávez LE. Integración docente-asistencial-investigativa (IDAI). Rev Cubana Educ Med Super 2000; 14(2):184-95.
10. Román O, Señoret SM. La relación docente-asistencial en el nuevo contexto que establece la reforma de salud. Rev Med Chil. 2007; 135(2): 251-256.

11. Vidal C, Quiñones J. Integración Docente-Asistencial. Educ Med Salud. 1986; 20, (1):458-465.

12. Pereira J, Aparecida L. La contribución de la articulación enseñanza y servicio para la construcción de la vigilancia de la salud: la perspectiva de los docentes. Rev Lat Am Enfermagem. 2009; 17(2):167173.

13. Agramonte A, Melón R, Pea A. Propuesta de guía metodológica para la formación de valores en los estudiantes de enfermería. Rev Cubana Enferm [Internet]. 2005; 21(2) [citado 13 enero 2011]. Disponible en: http://bvs.sld.cu/revistas/ enf/vol21_2_05/enf07205.htm

14. Chile, Ministerio de Salud. Norma general técnica y administrativa $\mathrm{N}^{\circ} 18$ sobre asignación y uso de los campos de formación profesional y técnica en el sistema nacional de servicios de salud y normas de protección para sus funcionarios, académicos, estudiantes y usuarios. Santiago de Chile: Ministerio de Salud; 2010.

15. Alva J, Verastegui G, Velásquez E, Pastor R, Moscoso B. Oferta y demanda de campos de práctica clínica para la formación de pregrado de estudiantes de ciencias de la salud en el Perú 20052009. Rev Peru Med Exp Salud Pública. 2011; 28 (2):194-201.

16. Colombia, Ministerio de Salud. Un nuevo paradigma en la relación entre la formación y los servicios de salud. Propuesta de estándares para la acreditación de los centros de formación en salud. Bogotá: Ministerio de Salud; 2002.

17. Vukusich A, Larrea R, Álamo M, Ponce JC, Valls G, González C, et al. Docencia de pregrado en medicina interna en un hospital privado: diez años de experiencia. Rev Med Chil. 2009; 137:1105-12. 
18. Lurie J, Mooney J. Relationship between clinical assessment and examination scores in determining clerkship grade. Med Educ. 2010; 44(2):177-83.

19. Brevis I, Sanhueza O. Integración docente asistencial en enfermería: problemas en su construcción? Rev. Eletrônica Enferm [Internet]. 2008; 10(2):367-73 [ci- tado 13 de enero 2011]. Disponible en: http://www.fen.ufg.br/revista/v10/n2/ v10n2a08.htm

20. MIDEPLAN. Encuesta de caracterización Socioeconómica de la Población chilena [Internet] [citado 20 enero 2011]. Disponible en: http://www.mideplan.cl/casen/Estadisticas/salud.html 